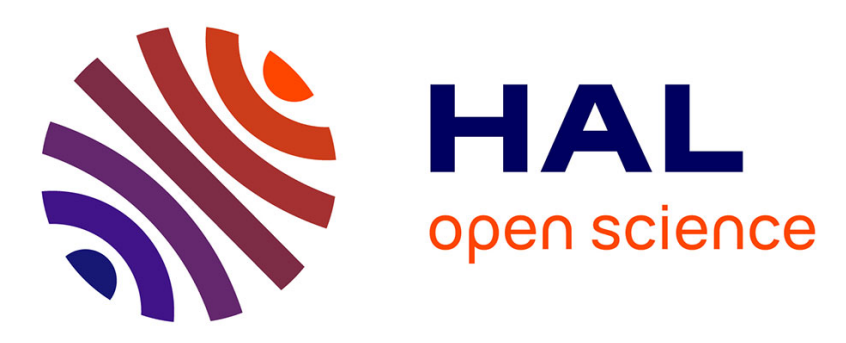

\title{
The double edged sword: The demographic consequences of the evolution of self-fertilization
}

Diala Abu Awad, Sylvain Billiard

\section{To cite this version:}

Diala Abu Awad, Sylvain Billiard. The double edged sword: The demographic consequences of the evolution of self-fertilization. Evolution - International Journal of Organic Evolution, 2017, 71 (5), pp.1178-1190. 10.1111/evo.13222 . hal-01525672

\section{HAL Id: hal-01525672 \\ https://hal.science/hal-01525672}

Submitted on 26 May 2020

HAL is a multi-disciplinary open access archive for the deposit and dissemination of scientific research documents, whether they are published or not. The documents may come from teaching and research institutions in France or abroad, or from public or private research centers.
L'archive ouverte pluridisciplinaire HAL, est destinée au dépôt et à la diffusion de documents scientifiques de niveau recherche, publiés ou non, émanant des établissements d'enseignement et de recherche français ou étrangers, des laboratoires publics ou privés. 


\title{
The double edged sword: The demographic consequences of the evolution of self-fertilisation
}

\author{
Diala Abu Awad ${ }^{1,2 *}$ and Sylvain Billiard ${ }^{1}$ \\ ${ }^{1}$ Univ. Lille, CNRS, UMR 8198 - Evo-Eco-Paleo, F-59000 Lille, France \\ ${ }^{2}$ INRA, UMR AGAP, 2 place Pierre Viala F-34060 Montpellier Cedex 1, \\ France
}

Phylogenies indicate that the transition from outcrossing to selfing is frequent, with selfing populations being more prone to extinction. The rates of transition to selfing and extinction, acting on different timescales, could explain the observed distributions of extant selfing species among taxa. However, phylogenetic and theoretical studies consider these mechanisms independently, i.e. transitions do not cause extinction. Here, we theoretically explore the demographic consequences of the evolution of self-fertilization. Deleterious mutations and mutations modifying the selfing rate are recurrently introduced and the number of offspring depends on individual fitness, allowing for a demographic feedback. We show that mutational meltdowns can be triggered in populations evolving near strict selfing. Populations having survived the demographic crash are more stable than ancestral outcrossing populations once deleterious mutations are purged. The relatively rapid time-scales in which extinctions occur indicate that during evolutionary transitions the accumulation of deleterious mutations may not be the cause of extinctions observed on longer time scales, which in turn could lead to the underestimation of transition rates from outcrossing to selfing.

Extinction, Inbreeding, Mating Systems, Models/Simulations, Mutations, Population Genetics

\begin{abstract}
This article has been accepted for publication and undergone full peer review but has not been through the copyediting, typesetting, pagination and proofreading process, which may lead to differences between this version and the Version of Record. Please cite this article as doi: 10.1111/evo.13222
\end{abstract}

This article is protected by copyright. All rights reserved. 


\section{Introduction}

The transition from predominantly outcrossing to self-fertilising reproductive systems is one of the most commonly observed and well documented of evolutionary transitions (Schoen et al., 1997; Goldberg et al., 2010; Igic and Busch, 2013; Barrett et al., 2014). The seeming uni-directionality of this evolutionary change (Igic and Busch, 2013) renders the relatively low frequency of predominant selfing in flowering plants $(\approx 11 \%$ Wright et al. 2013) somewhat paradoxical. Indeed, it has long been suggested that self-fertilisation is an evolutionary dead-end (Takebayashi and Morrell, 2001; Igic and Busch, 2013). Recent phylogenetic studies on diversification rates of flowering plants have supported this hypothesis. Results show lower net diversification rates of self-fertilising species compared to that of their outcrossing sister taxa due to higher extinction rates (Schoen et al., 1997; Goldberg et al., 2010; Igic and Busch, 2013). These phylogenetic results are supported by numerous theoretical investigations regarding the evolution of selfing and the effect of self-fertilization on the viability of populations. The genetic consequences of the evolution of self-fertilisation can be summarized by two main points: 1) The reduction of the effective population size (noted $N_{e}$, Wright et al. 2013) due to a reduced genetic diversity, which leads to weaker selection against deleterious mutations and weaker selection for beneficial mutations; and 2) The reduction of effective recombination and the increase of the HillRobertson effect (Kamran-Disfani and Agrawal, 2014). The combined effects of decreased genetic variation (i.e. lower adaptive potential, Glémin and Ronfort 2013) and reduced efficiency of recombination (leading to the accumulation of deleterious mutations, Lynch et al. 1995; Abu Awad et al. 2014) are expected to, in the long term, lead to the extinction of self-fertilising populations.

However, all these theoretical approaches suppose that the evolution from outcrossing to selfing and the extinction of selfing populations are independent, i.e. transitions from outcrossing to selfing do not affect population viability per se. On the one hand, existing models studying the extinction of self-fertilising populations have done so considering selfing as a fixed parameter and not a variable (Lynch et al., 1995; Abu Awad et al., 2014), while the change in reproductive systems can be relatively fast (Barrett et al., 2008;

${ }^{*}$ Corresponding author: E-mail: diala.abu-awad@inra.fr Address: INRA, UMR AGAP, 2 place Pierre Viala F-34060 Montpellier Cedex 1, France 
Shimizu and Tsuchimatsu, 2015), implying that the evolutionary transition and its ecological consequences can occur on the same timescale. On the other hand, works examining the evolution of mating systems showed that, providing that inbreeding depression is sufficiently low and that populations are constantly at their ecological and demographic equilibrium, an outcrossing population will always be invaded by a self-fertilising mutant (due to Fisher's automatic advantage, Fisher 1941; Charlesworth and Charlesworth 1987; Charlesworth et al. 1990; Cheptou and Dieckmann 2002; Porcher and Lande 2005). Any potential feed-back between the genetic and demographic states of populations during the transition from outcrossing to selfing is therefore neglected. This is especially problematic since we know that the evolution of self-fertilisation is accompanied by the purge of deleterious mutations (Charlesworth et al., 1990; Gervais et al., 2014), which may have demographic consequences. The question of whether the transition from outcrossing to selfing allows for the necessary demographic and genetic conditions for population extinction arises. If a viable outcrossing population evolves towards a selfing reproductive system is its probability of extinction increased? Or are only initially demographically unstable populations susceptible? It has been proposed that the purge of deleterious mutations can influence population size (Abu Awad et al., 2014). However, the speed at which the purge occurs may have different consequences: if it is very efficient then population size will immediately increase with the selfing rate; if it is not populations may suffer a decrease in size due to the introduction of inbred (hence less fit) individuals. Another unanswered question is whether or not initially outcrossing populations could naturally evolve to rates of self-fertilisation high enough to trigger a mutational meltdown (Barrett, 2010). To answer these questions, models considering the joint evolution of self-fertilisation, mutation load and population size are necessary.

Here we propose a model where recurrent deleterious mutations impact individual fitness (i.e. the reproductive rate) and consequently population size. Mutations promoting self-fertilisation are also introduced recurrently at a modifier locus in an initially outcrossing population at mutation-selectiondrift balance. As the genetic architecture and the strength of mutations affecting selfing rates seem to be variable (Barrett et al., 2008), we consider two modes of the evolution of self-fertilisation. We either continuously introduce mutations drawn in a distribution at the modifier locus so that selfing can evolve to any rate between 0 and 1 (Cheptou and Dieckmann, 2002; Epinat and Lenormand, 2009), or we consider the recurrent introduction of 
a single type of modifier with a fixed and strong effect on the selfing rate (Charlesworth et al., 1990; Gervais et al., 2014). We compare the two models for the evolution of self-fertilisation, noting their respective demographic and genetic consequences, such as the mutation load and effective relative census population size thus evaluating the importance of demographic feedback. Finally we explore the importance of demographic parameters such as the reproductive rate and the carrying capacity.

\section{Model}

Both the deterministic and simulation models stem from those presented in Abu Awad et al. (2014). We model a population of hermaphroditic individuals of varying size $N_{t}$, given by

$$
N_{t}=N_{t-1} R_{t-1}
$$

with $R_{t}$ the absolute fitness defined as

$$
R_{t}=r_{0}^{1-\frac{N_{t}}{K}} W_{t}
$$

where $r_{0}$ is the intrinsic reproductive rate, reproduction is assumed densitydependent ( $K$ is the carrying capacity) and the relative fitness $W_{t}$ at generation $t$ is assumed density-independent (Chevin and Lande, 2010). For an equilibrium relative fitness $W_{\text {det }}$ (i.e. at mutation-selection balance) and in the absence of demographic and genetic stochasticity, the deterministic population size $N_{\text {det }}$ is

$$
N_{\text {det }}=K\left(1+\frac{\operatorname{Ln}\left(W_{d e t}\right)}{\operatorname{Ln}\left(r_{0}\right)}\right) .
$$

A population with a relative fitness equal to or below $W_{d e t} \leq \frac{1}{r_{0}}$ is not viable as $N_{\text {det }} \leq 0$.

An approximation of the mean relative fitness $W_{\text {det }}$ at mutation-selection balance in a large population with an infinite number of selected loci can be obtained for a given set of demographic and genetic parameters (the haploid mutation rate $U$, the coefficient of selection $s$ and the dominance $h$, and the self-fertilisation rate $\alpha$ ) and taking into account the effects of linkage disequilibrium (Equation (11) from Roze (2015)):

$$
W_{\text {det }}=\left(1+I_{2}\right) \operatorname{Exp}\left[-U \frac{4 h+\alpha(1-4 h)}{2 h+\alpha(1-2 h)}\left(1+I_{1}\right)+\frac{2 \alpha}{2-\alpha} I_{2}\right]
$$

This article is protected by copyright. All rights reserved. 
with $I_{1}=2 U(1-h)(1-2 h) \frac{2+\alpha}{2-\alpha} T, I_{2}=U^{2}(1-2 h)^{2} T$ and $T=\frac{2 \alpha(1-\alpha)}{(4-\alpha)[2 h+\alpha(1-2 h)]^{2}}$.

Similarly, we use Roze's (2015) approximation for inbreeding depression with linkage disequilibrium (Equation (14) in Roze 2015) to evaluate for which genetic parameters self-fertilisation could evolve:

$$
\delta_{d e t}=1-\left(1+\frac{I_{2}}{4}\right) \operatorname{Exp}\left[-U \frac{1-2 h}{2 h+\alpha(1-2 h)}\left(1+I_{1}\right)+\frac{2 \alpha}{2-\alpha} I_{2}\right] .
$$

\subsection{Simulation Model}

In order to follow both the evolution of the selfing rate and its demographic consequences, we used an individual-centred model version of the deterministic model presented previously written in $\mathrm{C}++$ and available on Dryad. We consider a population with diploid hermaphroditic individuals and discrete non-overlapping generations, with a variable population size and a stable environment. The life cycle is as follows: mutation, selection, meiosis and reproduction. At a given time $t$, population size $N_{t}$ is given by

$$
N_{t}=\sum_{i=1}^{N_{t-1}} X_{t-1}^{i}
$$

where $X_{t}^{i}$ is the number of viable offspring an individual $i$ at time $t$ contributes via the female function to the next generation. $X_{t}^{i}$ is sampled from a Poisson distribution with mean $R_{t}^{i}=W_{t}^{i} r_{0}^{1-\frac{N_{t}}{K}}$ (the individual reproductive rate), where $W_{t}^{i}$ is the individual's relative fitness. Fitness is multiplicative and depends on the number of homozygous $\left(n_{h o}\right)$ and heterozygous $\left(n_{h e}\right)$ deleterious mutations, with a coefficient of selection $s$ and dominance $h$, carried by an individual $i$ given by

$$
W_{i}=(1-s)^{n_{h o}}(1-h s)^{n_{h e}} .
$$

The genetic properties of this model are modelled as in Roze (2009) and Abu Awad et al. (2014). We consider that each individual is represented by two homologous chromosomes of length $2 D$ with a potentially infinite number of loci. The map length is considered to be $D$ from the centre of the chromosome to the edge, hence representing a chromosome with a defined centromere. Near the centromere, we have included a modifier locus that affects the rate of self-fertilization as well as a neutral locus linked to 
the modifier. There are also two neutral loci integrated within the chromosome (at positions $-\frac{D}{2}$ and $\frac{D}{2}$ ) that recombine. Recombination occurs during gamete production and is considered to be uniform along the chromosome. New individuals are a combination of two gametes, either from two different individuals via outcrossing, or the same individual via selfing.

The number of new deleterious mutations occurring per chromosome per generation, is sampled from a Poisson distribution with mean $U$, where $2 U$ is the genomic mutation rate. Their position on the chromosome is sampled from a uniform distribution in $[-D, D]$. For simplicity we first consider that all deleterious mutations have the same coefficient of selection $s$ and dominance $h$. In order to evaluate the effect of the variance in mutational effects on fitness, certain parameter sets were run using a modified version of the script provided in Roze (2015), in which the coefficient of selection is drawn from a log-normal distribution (with mean $\mu$ and variance $\sigma^{2}$ and density function $\phi(s)=\exp \left[-(\log (s)-\mu)^{2} /(2 \sigma)^{2}\right] /(s \sigma \sqrt{2 \pi})$, truncated at $s=1$ (an approximation of Fisher's geometric landscape model). We assume that the heterozygous effect $\eta$ is constant, making $s$ and $h$ negatively correlated (i.e very deleterious mutations are recessive). The distribution of the dominance coefficients $(h=\eta / s)$ is given by $\psi(h)=\left(\eta / h^{2}\right) \phi(\eta / h)$. Self-fertilisation of an ovule produced by individual $i$ occurs with probability $\alpha_{t}^{i}$ and is given by

$$
\alpha_{t}^{i}=\frac{\alpha_{0} W_{t}^{i}}{\alpha_{0} W_{t}^{i}+\left(1-\alpha_{0}\right) \frac{\sum_{j \neq i} W_{t}^{j}}{N_{t}-1}},
$$

where $\alpha_{0}$ is the proportion of an individual's male gametes that are available for self-fertilization. The probability of self-fertilization depends on the individual's relative fitness $W_{t}^{i}$ compared to the average relative fitness of the other possible fathers in the population $\left(\frac{\sum_{j \neq i} W_{t}^{i}}{N_{t}-1}\right)$. The lower an individual's relative fitness as a father, the lower the proportion of offspring produced via selfing. The proportion of an individual's offspring produced by selffertilization is sampled from a binomial distribution with parameters $\alpha_{t}^{i}$ and $X_{t}^{i}$. When $\alpha_{0}=0$ the population is strictly outcrossing and the population is automatically considered non-viable if $N_{t}<2$. When outcrossing occurs, a father, say individual $j$, is randomly chosen among all possible individuals, except the focal individual to be sired. It successfully sires the focal individual with probability $W_{j}$. A father is drawn until fertilization is successful, hence there is no limitation in the availability of male gametes. 
We explore two modes of transition from outcrossing to a self-fertilising reproductive regime by introducing one of two types of modifiers: 1) The rate of selfing of a new allele introduced at the modifier locus via mutation is drawn from a uniform distribution in $\left[\alpha_{0}-d, \alpha_{0}+d\right]$, i.e. centred around the self-fertilisation rate $\alpha_{0}$ (Equation (8)) of the mutant's parent, i,e. the new allele inducing a change in the selfing rate $\alpha_{0}$ of mean 0 and variance $\frac{d^{2}}{3}$. In this mode of transition, the rate of self-fertilisation can evolve anywhere between 0 and 1 until it reaches an Evolutionary Stable Strategy (ESS); and 2) In order to estimate the effect of the selfing rate induced by mutations at the modifier locus an allele resulting in a fixed selfing rate $\alpha_{m}$ is introduced recurrently, with no back mutations, thus only increasing the selfing rate. In both models, alleles at the modifiers locus are co-dominant.

\subsubsection{Initial conditions, measured variables and parameters run}

Populations start at size $K$, with no deleterious mutations and monomorphic neutral loci. Deleterious and neutral mutations are then introduced and simulations are run until the population reaches mutation-selection-drift balance or goes extinct. Mutation-selection-drift balance is assumed to be reached when mean relative fitness $\bar{W}_{t}$ over one thousand generations varies by less than 1 per cent compared to the average $\bar{W}_{t}$ of the previous thousand generations. Once the equilibrium is reached, mutations are introduced at the modifier locus. Simulations are then run until population extinction or, once a new equilibrium is reached (the selfing rate and fitness become stable), for at least a further 100 thousand generations. So as to determine the genetic and demographic properties (e.g. the probability of extinction) of fully outcrossing populations, as well as to evaluate consequences of the selfing rate versus the consequences of the transition from outcrossing to a given selfing rate, simulations in which no mutations are introduced at the selfing modifier locus are also run for 150 thousand generations.

Population size, mean fitness (using Equation (7)), inbreeding depression, effective population size and the effective rate of self-fertilisation are measured each generation. The mean and standard deviation of all these variables are estimated (conditioned on survival of the population) over one thousand independent replicates for each parameter set. Inbreeding depression $\delta_{t}$ is estimated by generating one selfed and one outcrossed offspring from a sample of 200 individuals. If the population size $N_{t}$ is smaller than 200 then the entire population is sampled. In order to estimate the effective 
population size $N_{e}$, as we consider that the neutral loci follow an infinite allele model, we use the expression for the expected mean frequency of heterozygotes $H_{e}$ using the infinite alleles model in a population of effective size $N_{e}$ (Gale, 1990, p. 325) :

$$
H_{e}=\frac{4 \mu N_{e}}{1+4 \mu N_{e}}
$$

where $\mu$ is the mutation rate at the locus considered. From our simulations, we can calculate $H_{e}$ at each neutral locus using

$$
H_{e}=1-\sum_{i=1}^{n a} p_{i}^{2}
$$

(with na the number of alleles segregating at the neutral locus and $p_{i}$ the frequency of allele $i$ in the population at that locus). We also measure the diversity at the neutral locus linked to the modifier to asses the contribution of the recurrent introduction of mutations at the modifier of the self-fertilisation rate on the observed $N_{e}$.

We ran simulations for intrinsic reproductive rates $r_{0}=2,4$ and 10, with a haploid genomic rate $U$ between 0.1 and 0.3 for $r_{0}=2$ (as for higher values of $U$ all populations go extinct) and $U$ between 0.1 and 0.6 for the other values of $r_{0}$. Mutations in simulations run with fixed values of $s$ and $h$ were either mildly deleterious or very deleterious (coefficient of selection $s=0.02$ and 0.2 ), and with either completely recessive with $h=0$ or moderately recessive $(h=0.1$ and 0.2$)$. In simulations where the coefficient of selection of new mutations is sampled from a log-normal distribution, as in Roze (2015) the mean and variance of the distribution are $\approx-3.316$ and 0.8 respectively, resulting in a mean coefficient of selection $s=0.05$ and $h=0.25$ (the mean of the product $s h$ is therefore $\eta \approx 0.00659)$. The recombination rate $D$ is set at 10, as $D$ over 10 has very little effect on the results (Roze, 2012), even though selective interference is expected when there is selfing (Roze, 2015). The mutation at the neutral loci is set at $10^{-3}$. At the modifier locus, two mutation rates are simulated, $10^{-3}$ and $10^{-5}$. For the evolution of self-fertilisation to an ESS (mutations are drawn from a uniform distribution $\left.\left[\alpha_{0}-d, \alpha_{0}+d\right]\right)$, simulations were run with new alleles introduced at the modifier locus having an effect range $d$ of $0.01,0.1,0.5$ and 0.9 (giving means around the initial selfing rate of 0 and variances $3.10^{-4}, 3.10^{-3}, 0.08$ and 0.27 respectively). When selfing evolves due to a single type of mutant allele 
at the modifier locus with selfing rate $\alpha_{m}$, simulations are run for different $\alpha_{m}=0.1,0.3,0.6,0.9,0.95$ and 0.99. Simulations without the evolution of selfing are run for fully outcrossing populations $\left(\alpha_{0}=0\right)$ and values $\alpha_{0}$ set at the same values as those used for $\alpha_{m}$.

\section{Results}

Our goal is to investigate the demographic consequences of the evolution of self-fertilisation under different scenarios. First, we explore the expected demographic and genetic properties of populations without the evolution of self-fertilisation, notably population size (which directly reflects population fitness, see Equations (3) and (6)) and inbreeding depression. We compare simulation results with analytical expectations detailed in the previous section and from previous theoretical works (Roze, 2015). We then allow the evolution of self-fertilisation using two approaches: 1) Self-fertilisation is allowed to evolve freely until it reaches an Evolutionary Stable Strategy (ESS) and 2) A modifier with a large fixed effect is introduced recurrently into the population and can even go to fixation. Finally we touch on the evolution of the effective population size during and after the transition from an outcrossing to a self-fertilising reproductive system and discuss the effects of the demographic parameters (the intrinsic reproductive rate $r_{0}$ and the carrying capacity $K$ ) on population persistence.

We briefly summarise the results obtained from simulations for different parameter sets in Table 1, taking into account the initial (before the evolution of selfing) and final (after the evolution of selfing) states of populations depending on the genetic and demographic parameters. Initial outcrossing populations are either stable or unstable: Stable populations are those which are not expected to go extinct in a relatively short time frame as all simulations result in viable populations for at least 150 thousand generations. Unstable populations are those susceptible to extinction due to demographic and genetic stochasticity within a relatively short time frame (at least one out of one thousand simulations resulted in extinction).

\subsection{Fixed self-fertilisation rates}

In Figure 1 we show that the expected inbreeding depression and population size at mutation-selection equilibrium for populations with fixed rates 
of self-fertilisation are well predicted by the analytical terms in Equations $(3),(4)$ and (5). When mutations are completely recessive $(h=0$, see Figure 1a), inbreeding depression is much higher than 0.5 and self-fertilisation is not expected to evolve in such populations. With partially recessive mutations, inbreeding depression can be low enough to allow the evolution of selfing, provided the deleterious mutation rate is small enough $(U \leq 0.2$ for $h=0.1$ and $U \leq 0.5$ for $h=0.2$ ). For levels of inbreeding depression slightly higher than 0.5 , the purging provoked by the recurrent introduction of selfing modifiers, as well as the consequences of genetic drift in small populations, could facilitate the evolution of self-fertilisation.

In Figure 1b, we show that population size increases with the selfing rate (as deleterious mutations are better purged) and decreases with the deleterious mutation rate $U$. If the deleterious mutation rate is too high, or the reproductive rate too low, populations may be non-viable (the expected population size at equilibrium $N_{\text {det }}$ is negative). From Figure 1b, one can expect that population size should increase as the mean selfing rate increases during the transition from outcrossing to selfing because of purging, but only if the demographic effects of purging are negligible, which, as we show below, is not always the case. However, even if the effects of purging are negligible, if the coefficient of selection is too small (i.e. $s=0.02$ ), populations that evolve to very high selfing rates are expected to go extinct as all simulations run for a selfing rate $\alpha \geq 0.98$ and $s=0.02$ resulted in extinction (even for low deleterious mutation rates and high intrinsic reproductive rates).

\subsection{Evolution of selfing rates to the ESS}

We investigate the ESS of the selfing rate by introducing mutations at a selfing modifier locus (that can either increase or decrease the selfing rate) in an initially fully outcrossing population. The effects of these mutations are drawn from a uniform distribution centred around the modifier's current selfing rate. New alleles introduced at the modifier locus can therefore either decrease or increase the self-fertilisation rate. We follow the evolution of self-fertilisation until we either reach an ESS (defined as the stabilisation of both the self-fertilisation rate and population fitness) or the population goes to extinction.

In populations where self-fertilisation evolves $(\delta$ is sufficiently low, i.e. $U$ is not too high and $h>0$, see Figure 1), the observed rate of self-fertilisation is not far from the expected ESS of selfing rate $\alpha=1$ (the mean observed 
selfing rate $\bar{\alpha} \approx 0.98$, Figure 2 ). The coefficient of selection $s$ influences the time needed for populations to purge their deleterious load and reach the ESS, as well as the viability of populations once the ESS value of selfing is reached (Figure 2a). In the first phase, population size (hence fitness) increases and inbreeding depression decreases with the selfing rate. Once the ESS of the selfing rate is attained, populations either remain viable, or, if the coefficient of selection is small (i.e $s=0.02$ ), deleterious mutations accumulate (Supplementary Figure ??), leading to extinction (bottom frame of Figure 2a). Extinction occurs relatively fast once the ESS of the selfing rate is reached, with the average time to extinction being situated between 2000 and 40000 generations depending on the parameter set considered (for the parameter set shown in Figure 2, extinction occurs within 2500 generations for $s=0.02)$. As expected from the simulations run with a fixed selfing rate $\alpha_{0}=0.99$ (Figure $1 \mathrm{~b}$ ), eventually all populations in which deleterious mutations have a small coefficient of selection go extinct after the evolution of high selfing rates. This remains true for simulations run with a very small effect range of mutations at the selfing modifier locus $(d=0.01)$ and also in the case of a coefficient of selection of deleterious mutations sampled from a distribution, with mean $s=0.05$ and $h=0.25$ (even for low deleterious mutation rates and high intrinsic reproductive rates, Supplementary Figure ??). Decreasing the mutation rate at the modifier locus $\left(\mu=10^{-5}\right.$, Supplementary Figure ??) increases the time necessary for populations to evolve selfing rates high enough to trigger the accumulation of deleterious mutations, but has no effect on the probability of extinction (i.e. all populations go extinct). Once again, when high selfing rates evolve, time to extinction is relatively short.

In Figure 2b we examine how the change of the selfing rate affects population genetics in comparison to expectations from population genetics models. More specifically we follow the changes in mean population fitness and inbreeding depression conditioned on survival change, not as a function of time, but of the mean selfing rate $\bar{\alpha}_{t}$. When compared to the expected relationship between the rate of self-fertilisation and these two variables (fitness and inbreeding depression) under the model with infinite loci and linkage disequilibrium (Equations (4) and (5)), we find that as the rate of self-fertilisation increases, the genetic properties of populations are well predicted by the analytical expressions from Equations (4) and (5) (represented by the line with the black contour in Figure $2 \mathrm{~b}$ ), except for $s=0.02$ where fitness plunges at high selfing rates. 
Despite the recurrent introduction of mutations at the selfing modifier locus that could decrease the selfing rate, these do not go to fixation, hence implying that the evolution to high selfing rates is irreversible. The same patterns are observed for mutations of a larger range of effects at the modifier (greater values of $d$, Supplementary Figure ??), the main differences being a faster evolution to the ESS and, for mildly deleterious mutations (i.e $s=$ 0.02), shorter times to extinction due to the accumulation of deleterious mutations once at the ESS or extinction due to the demographic consequences of the purging of deleterious alleles (discussed below).

\subsection{Modifiers with large fixed effect}

In the previous section, self-fertilisation was allowed to evolve freely and continuously towards the ESS. However, as it is possible that selfing can evolve from a single effect mutation, we also explore the consequences of introducing recurrent mutations of a strong effect at the selfing modifier locus. The consequences of the introduction of such mutations depends on the demographic state of the initial outcrossing population, as different parameter sets can lead to either stable or unstable populations (Table 1). We define the former as populations that are part of a parameter set for which all simulations result in viable populations in the observed time frame and the latter are populations that are part of a parameter set in which at least one population out of the 1000 simulated goes extinct before 150 thousand generations due to demographic stochasticity (and not parameter sets for which all populations go extinct).

As predicted in Figure 1b, population size at equilibrium is higher once the alleles increasing the selfing rate are fixed at the modifier locus due to a lower mutational load (hence higher population fitness) than in the initial ouctrossing population. In simulations run with $s=0.02$ population size does not immediately increase as the selfing rate increases, but there is an initial dip (resp. peak) in population size (resp. inbreeding depression, see Figure 3). This leads to an initial deviation from analytical expectations regarding the relationship between self-fertilisation and the genetic properties of populations that is accentuated by the mutants' selfing rate $\alpha_{m}$ (Figure $3 \mathrm{~b}$ ). This pattern (dip and peak of fitness) is not observed for $s=0.2$ (Supplementary Figure ??).

The dip in population size is due to the demographic consequences of the purge: as individuals self-fertilise, they produce a portion of non-viable off- 
spring carrying deleterious mutations at the homozygous state. As the selfing rate increases, this portion of non-viable offspring increases, further decreasing the reproductive rate and hence population size, until the mutations have been purged and the population is at mutation-selection equilibrium, after which populations go to their equilibrium size. A temporary increase of inbreeding depression could be explained by two phenomena: the purging of deleterious mutations and the potentially independent origins of selfing lineages. The latter hypothesis seems to contribute little to this observation, as the amplitude of the peak in the level of inbreeding depression is not greatly affected by the mutation rates at the modifier locus and results indicate that selfing lineages probably originate from a single self-fertilising ancestor (Supplementary Figure ??). Under the first hypothesis purging has a tendency to eliminate inbred lineages that become highly homozygous for deleterious alleles, while conserving those with higher heterozygosity than initially present in the population. A consequence of this increased heterozygosity is an increase in inbreeding depression and a higher short-term diversity, as confirmed by the observed evolution of neutral genetic diversity (see section below).

During the purging phase, unstable populations can go extinct (Figure 3c) before the fixation of the self-fertilising allele at the modifier locus. The higher the selfing rate of the mutant $\alpha_{m}$, the stronger the initial dip in population size and the higher the extinction rate in a relatively short time frame (in less than 500 generations, see Figure 3c). The mutation rate at the modifier locus has no effect on the amplitude of the dip in population size, nor on the proportion of populations that go extinct during the purging phase (Supplementary Figure ??). Roughly, there are two outcomes: populations suffering less drastic bottlenecks tend to recover and reach the new equilibrium size, whereas those with smaller sizes go to extinction. Once the new equilibrium has been reached, contrary to their unstable initial state, populations become stable, with no extinctions observed for at least 150 thousand generations (populations run with $\alpha_{0}=\alpha_{m}$ are also stable on the long-term). The transition towards higher selfing rates has thus two contradictory effects: it decreases the survival rate of unstable populations on the short term, however populations that survive the demographic consequences of the purge go on to be stable on the long term, resulting in an evolutionary rescue. If however after this initial fixation of a self-fertilising modifier is followed by an evolution of self-fertilisation to the ESS (with smaller modifier effects), we should expect that these populations would go to inevitable extinction 
(as shown in the previous section). Indeed, when the range of the effects of the selfing modifier are large $(d>0.1)$, we also observe the dip in population size and in some cases extinction as for modifiers with a fixed effect (Supplementary Figure ??).

\subsection{Effective population size and lineage selection}

In Figure 4, we follow the changes in effective population size relative to demographic size $\left(N_{e} / N\right)$ using the recombinant neutral loci. With selfing modifiers of a fixed effect and mildly deleterious mutations $(s=0.02)$ we can observe three phases in the changes of the ratio $N_{e} / N$ (Figure 4): An initial peak followed by a rapid dip and finally a slow increase to the new equilibrium ratio (lower than that observed in the initial outcrossing population). These phases are accentuated by the amplitude of the effect of the alleles introduced at the selfing modifier locus (the range $d$ in the case of continuous evolution, and $\alpha_{m}$ of the fixed effect mutations). If self-fertilisation evolves continuously with small mutations at the modifier locus, this peak is never observed and increasing self-fertilisation leads to an almost linear decrease of the $\frac{N_{e}}{N}$ ratio (Supplementary Figure ??).

The initial peak of $N_{e} / N$ corresponds to the dip in population size and peak in inbreeding depression (see Figure 3c). This implies that the demographic consequences of the purge occur at a faster pace than the decrease in genetic variability. This indicates that this peak phase is in large part due to the purge of homozygous deleterious mutations, initially leaving an excess of heterozygous individuals (thus increasing inbreeding depression, Figure 3). As population size recovers, the ratio $\frac{N_{e}}{N}$ decreases to below its initial value, once again because of the differences in time scales necessary for demographic and genetic equilibria, then increases slowly to its new equilibrium value. New equilibrium population size is therefore reached before that of neutral diversity, as expected from works exploring the time necessary for the recovery of neutral diversity after the re-establishment of a large population size following a demographic bottleneck (Tajima, 1989). Indeed this pattern is not observed when the purge is not accompanied by a demographic bottleneck (i.e. for low values of $d$ and for $s=0.2$, Supplementary Figure $? ?)$. 


\subsection{Demographic parameters and their consequences}

Extinctions during the transition from outcrossing to self-fertilisation occur only in initially unstable populations (see Table 1). In order to verify whether these extinctions are a consequence of small population size, simulations for the same genetic parameters and similar initial population sizes of outcrossing populations (i.e higher reproductive rates and lower carrying capacities) were run. Figure 5 shows that, in spite of the dip in population size due to the demographic consequences of the purge, no populations go extinct when the reproductive rate is higher. Population size per se is therefore not a deciding factor in the persistence probability of a population during a mating system transition. This implies that extinction is a consequence of the transition from outcrossing to a selfing reproductive system coupled with small reproductive rates.

\section{Discussion}

Models on the evolution of self-fertilisation have so far not taken into account the potential consequences of transition from outcrossing to selfing on population demography as well as any feedback between population size and selection. We first discuss to what extent demographic feedback can affect the evolution of self-fertilisation. Then we examine the mechanisms through which self-fertilisation can lead to population extinction both on the short and long-term. Third, we compare our results to existing empirical data on the evolution of self-fertilisation.

\subsection{Final states are unchanged by demographic feed- back in viable populations}

The equilibrium states (i.e. mutation-selection-drift balance) of viable populations that have successfully evolved from an outcrossing to a self-fertilising reproductive system agree with the expected equilibria of self-fertilising populations as in previous models without demographic feedback (Figures $2 \mathrm{~b}$ and 3b, Lande and Schemske, 1985; Charlesworth et al., 1990; Kamran-Disfani and Agrawal, 2014; Roze, 2015). As expected, in order for self-fertilisation to evolve, inbreeding depression must be low enough and the purging of deleterious mutations must be possible. Selection can either facilitate the purge 
of deleterious mutations, and (in the case of the continuous evolution of selfing) populations evolve very high rates of self-fertilisation $(\approx 1)$, or deleterious mutations cannot be purged and the population remains outcrossing (Charlesworth et al., 1990; Gervais et al., 2014).

We generally find that if the rate of self-fertilisation were to evolve continuously with small enough mutational steps, the continuous evolution of selfing results in a smooth and continuous change of the genetic and demographic variables and analytical theory provides relatively good predictions of how the genetic variables evolve with the change of self-fertilisation (except for small $s$ when high selfing leads to extinction Figure $2 \mathrm{~b}$ ). In this case, the rate of selfing at a time $t$ can be used to predict population fitness (hence size) as well as the level of inbreeding depression (Figure 3). However, for mildly deleterious mutations $(s=0.02)$ and modifiers of stronger effects on the selfing rate (be it the continuous evolution to the ESS or mutation to a fixed rate of self-fertilisation, with parameter $\alpha_{m}$ ), there is an initial increase (resp. decrease) in inbreeding depression (resp. fitness and size, see Figure 3) before the population reaches its equilibrium state.

The initial increase of inbreeding depression is not observed when selfing evolves slowly as the purge occurs at the same speed as the change in the level of inbreeding. However, this phenomenon is intuitive as the selection against individuals homozygous for deleterious alleles increases the population's heterozygosity, thus resulting in higher inbreeding depression. High inbreeding depression in selfing populations may therefore be an indicator of a recent shift in the reproductive regime. This is further accentuated by the potential existence of several selfing lineages with different mutational backgrounds, which increases the variability between individuals. Independent appearances of alleles promoting selfing rate have been documented in natural populations (Shimizu and Tsuchimatsu, 2015), such phenomena during the transition from outcrossing are therefore plausible. A snowball effect could also be in play, with one allele increasing in frequency, triggering the purge, hence allowing the increase in frequency of any other alleles segregating at the modifier locus (see Supplementary Figure ??b).

\subsection{Towards evolutionary suicide}

As suggested both by theoretical and empirical works (Takebayashi and Morrell, 2001; Wright et al., 2013), self-fertilisation can be an evolutionary deadend. Theoretical works have pointed to the accumulation of mildly delete- 
rious mutations in populations with high selfing rates being responsible for population extinction (Lynch et al., 1995; Abu Awad et al., 2014), which seems to be the case even when the effects of deleterious mutations are sampled from a distribution (Supplementary Figure 2b). However, these results depend greatly on the properties of new deleterious mutations, requiring them to have, on average, small selective coefficients, which is supported by empirical estimations of the distribution of mutational effects on fitness (Agrawal and Whitlock, 2011; Keightley, 2012). Here we show that high rates of self-fertilisation are not only an evolutionary dead-end but a mechanism for evolutionary suicide. For this, three conditions must be fulfilled: 1) Ancestral outcrossing populations must be viable, 2) The evolution from outcrossing to selfing must be possible (inbreeding depression in the initial population must be low enough) and 3) The equilibrium rates of self-fertilisation attained must be high enough to trigger a mutational melt-down. Figure 2 shows that all these conditions can indeed be fulfilled. Though it has also previously been shown that interspecific interactions can cause evolutionary suicide of selfing populations (Lepers et al., 2014) due to feedback mechanisms between demography and genetics, our work is the first to explicitly show that even without such complex interactions initially stable populations continuously evolving from outcrossing to very high self-fertilisation rates can go to extinction.

\subsection{Purging: the up and the down side}

For mating systems to evolve to strict selfing, deleterious mutations must be purged, and when this is possible the final state of populations is either longterm stability or relatively rapid extinction due to either the demographic consequences of the purge or, when selfing rates are very high, a mutational meltdown is triggered. Though purging is often considered to be a positive mechanism in the evolution of selfing, especially since it is expected to decrease inbreeding depression (Charlesworth et al., 1990; Frankham et al., 2001), any potential demographic consequences of expressing deleterious mutations during purging through inbreeding can be important and be the cause of population extinction as shown empirically in Drosophila melanogaster (Frankham et al., 2001). The strength of the purge depends not only on the properties of deleterious mutations but also on the strength of mutations modifying the selfing rate: The smaller the effect of deleterious mutations and the higher the selfing rate of individuals carrying mutations at the self- 
ing modifier locus, the stronger the strength of the purge, and, despite the important accompanying decrease in inbreeding depression, the greater the probability of extinction during the transition (see Figure 3c).

Wright and Barrett (2010) have suggested that the high extinction rates observed in populations that have recently transitioned to a self-fertilising reproductive system could be due to the unfavourable demographic properties of such populations and not the reproductive system as such. Our results support this hypothesis, as in unstable populations the introduction of mutations at the modifier locus precipitates the extinction of populations that are already at a high risk of extinction because of demographic stochasticity. On the other hand, populations surviving this purge, are then stable, in which case the evolution of self-fertilisation can be quantified as a mechanism of evolutionary rescue (Figure 3c). However, this remains true provided populations do not evolve to extremely high rates of self-fertilisation, triggering a mutational meltdown in a relatively short time compared to the initially outcrossing populations.

Population bottlenecks are commonly accompanied with a decrease in neutral genetic diversity (or effective population size $N_{e}$, Frankham et al. 2002). The decrease in population size brought on by the purge of mildly deleterious mutations $(s=0.02)$ is associated with an increased $\frac{N_{e}}{N}$ ratio. When it is small, this ratio is often viewed as a signal for population vulnerability in conservation biology (Frankham et al., 2014), but as pointed out by Palstra and Fraser (2012), an $\frac{N_{e}}{N}$ ratio higher than 1 can be associated with a population with non-constant size. Indeed, the high $\frac{N_{e}}{N}$ ratio from our simulations is associated with the decline in population size during the purging phase. The peak in $\frac{N_{e}}{N}$ indicates that population size declines at a different scale than the neutral genetic diversity. Counter-intuitively, a recent transition to selfing, or the increasing frequency of a self-fertilising genotype (Figure 4 and Supplementary Figure ??), will result in a high $\frac{N_{e}}{N}$ and in some cases may in fact be a signal of demographic vulnerability due to decreasing population size.

The demographic and genetic consequences of the transition from outcrossing to selfing may be reduced if we were to take into account the consequences of sib-mating on the frequencies and states of segregating deleterious mutations, as suggested by Porcher and Lande (2016). However, taking sibmating into account would require a spatially structured model and would be applicable only to species in which dispersion is limited. 


\subsection{Implications for macro-evolution}

Self-fertilisation has been considered an evolutionary dead-end for at least half a century (Stebbins, 1957). This has been supported by empirical works, for instance in the Solonaceae, where self-fertilising species have been shown to have higher extinction rates than their self-incompatible sister species (Goldberg et al., 2010). Higher extinction rates have been attributed to two major phenomena: 1) The lack of adaptive potential due to lower standing variation in selfing species Glémin and Ronfort 2013) and 2) The accumulation of deleterious mutations, leading to the mutational meltdown (Lynch et al., 1995; Abu Awad et al., 2014). Our results support that the transition from an outcrossing to a selfing reproductive system is associated with higher extinction rates due to the accumulation of deleterious mutations. Also, due to the feedback between population size and selection, populations can become vulnerable during the transition from outcrossing, further increasing the probability of extinction. However, the evolution of self-fertilisation is a mechanism that can increase population viability as it is expected to increase population mean fitness, hence population size. This should render populations less susceptible to extinction, all the more so in the presence of ecological perturbations (Thomann et al., 2013).

The rapidity with which extinction occurs due to the consequences of deleterious mutations leads us to conclude that the extinctions observed on the time scales on which phylogenies are based may not be due to mutational meltdowns but to a lack of adaptive potential as would be expected from the very small effective population sizes $N_{e}$ of selfing populations (Figure 4 and Supplementary Figure ??, Stebbins 1957; Takebayashi and Morrell 2001; Glémin and Ronfort 2013). Contemporary selfing species are therefore most probably those that have survived the initial purging process and which possess genetic mechanisms that allow the avoidance of long-term fitness decline as suggested by Wright et al. (2008) (i.e. in our model if deleterious mutations were to have stronger coefficients of selection, then a low effective population size would not result in triggering a mutational meltdown, Supplementary Figure ??b). However our results have implications for the transition rates used in works on plant phylogenies (Goldberg et al., 2010; Goldberg and Igic, 2012), which may indeed be underestimated as such short lived transitions would not be easily detected.

Finally, we find that the populations most robust to the transition from outcrossing to selfing are, for a same initial population size, those with a 
higher reproductive rate (Figure 5). This implies that selfing may be associated with high reproductive rates relative to those observed in outcrossing sister species and/or populations. $r$-strategies have indeed been observed in natural populations, with for instance Arabidopsis thaliana having reproductive rates that are several orders of magnitude larger than outcrossing sister taxa (Van Daele et al., 2012) and self-fertilisation being associated with an annual life history (Morgan et al., 1997). Our results therefore support that if the selfing lineages currently observed generally follow $r$-strategies, it may be because of lineage selection: i.e. lineages have a higher probability of surviving the transition from outcrossing to selfing when their reproductive output is high.

\section{Acknowledgements}

We would like to thank Denis Roze for many helpful discussions. Numerical results presented in this paper were carried out using France Grille, CNRS and on the Biomed virtual organization of the European Grid Infrastructure (http://www.egi.eu) via DIRAC (http://diracgrid.org). We thank the technical staff of the European Grid Infrastructure and the supporting National Grid Initiatives for providing the technical support and infrastructure. This work was supported by the French Agence Nationale de la Recherche ANR11-BSV7- 013-03 and benefited from the support of the Chair Modlisation Mathmatique et Biodiversit of Veolia Environnement - cole Polytechnique Musum National dHistoire Naturelle - Fondation X.

\section{References}

Abu Awad, D., S. Gallina, C. Bonamy, and S. Billiard, 2014. The Interaction between Selection, Demography and Selfing and How It Affects Population Viability (vol 9, e86125, 2014). PLOS ONE 9.

Agrawal, A. and M. Whitlock, 2011. Inferences about the distribution of dominance drawn from yeast gene knockout data. Genetics 187:553.

Barrett, S., 2010. Understanding plant reproductive diversity. Philos Trans R Soc Lond B Biol Sci 365:99-109. 
Barrett, S., R. Arunkumar, and S. Wright, 2014. The demography and population genomics of evolutionary transitions to self-fertilization in plants. Philos Trans R Soc Lond B Biol Sci 369.

Barrett, S., R. Colautti, and C. Eckert, 2008. Plant reproductive systems and evolution during biological invasion. Mol Ecol 17:373-383.

Charlesworth, D. and B. Charlesworth, 1987. Inbreeding depression and its evolutionary consequences. Annu Rev Ecol Syst 18:237-268.

Charlesworth, D., M. Morgan, and B. Charlesworth, 1990. Inbreeding depression, genetic load, and the evolution of outcrossing rates in a multilocus system with no linkage. Evolution 44:1469-1489.

Cheptou, P. O. and U. Dieckmann, 2002. The evolution of self-fertilization in density-regulated populations. Proc R Soc Lond B Biol Sci 269:1177-1186.

Chevin, L. and R. Lande, 2010. When do adaptive plasticity and genetic evolution prevent extinction of a density-regulated population? Evolution 64:1143-1150.

Epinat, G. and T. Lenormand, 2009. The evolution of assortative mating and selfing with in- and outbreeding depression. Evolution 63:2047-2060.

Fisher, R., 1941. Average excess and average effect of a gene substitution. Ann Eugenic 11:53-63.

Frankham, R., J. D. Ballou, and D. A. Briscoe, 2002. Introduction to Conservation Genetics:. Cambridge University Press, Cambridge.

Frankham, R., C. Bradshaw, and B. Brook, 2014. Genetics in conservation management: Revised recommendations for the 50/500 rules, Red List criteria and population viability analyses. Biol Conserv 170:56-63.

Frankham, R., D. Gilligan, D. Morris, and D. Briscoe, 2001. Inbreeding and extinction: Effects of purging. Conserv. Genet. 2:279-285.

Gale, J., 1990. Theoretical population genetics. Unwin Hyman.

Gervais, C., D. Abu Awad, D. Roze, V. Castric, and S. Billiard, 2014. Genetic architecture of inbreeding depression and the maintenance of gametophytic self-incompatibility. Evolution 68:3317-3324. 
Glémin, S. and J. Ronfort, 2013. Adaptation and maladaptation in selfing and outcrossing species: new mutations versus standing variation. Evolution 67:225-240.

Goldberg, E. and B. Igic, 2012. Tempo and mode in plant breeding system evolution. Evolution 66:3701-3709.

Goldberg, E., J. Kohn, R. Lande, K. Robertson, S. Smith, and B. Igic, 2010. Species selection maintains self-incompatibility. Science 330:493-495.

Igic, B. and J. Busch, 2013. Is self-fertilization an evolutionary dead end? New Phytol 198:386-397.

Kamran-Disfani, A. and A. F. Agrawal, 2014. Selfing, adaptation and background selection in finite populations. J Evol Biol 27:1360-1371.

Keightley, P., 2012. Rates and Fitness Consequences of New Mutations in Humans. Genetics 190:295-304.

Lande, R. and D. Schemske, 1985. The evolution of self-fertilization and inbreeding depression in plants .1. genetic models. Evolution 39:24-40.

Lepers, C., M. Dufay, and S. Billiard, 2014. How does pollination mutualism affect the evolution of prior self-fertilization? A model. Evolution 68:35813598.

Lynch, M., J. Conery, and R. Burger, 1995. Mutational meltdowns in sexual populations. Evolution 49:1067-1080.

Morgan, M., D. Schoen, and T. Bataillon, 1997. The evolution of selffertilization in perennials. Am Nat 150:618-638.

Palstra, F. and D. Fraser, 2012. Effective/census population size ratio estimation: a compendium and appraisal. Ecol Evol 2:2357-2365.

Porcher, E. and R. Lande, 2005. Loss of gametophytic self-incompatibility with evolution of inbreeding depression. Evolution 59:46-60.

- 2016. Inbreeding depression under mixed outcrossing, selffertilization and sib-mating. BMC Evol Biol 16:105. 
Roze, D., 2009. Diploidy, Population Structure, and the Evolution of Recombination. Am Nat 174:S79-S94.

— 2012 . Spatial heterogeneity in the strength of selection against deleterious alleles and the mutation load. Heredity 109:137-145.

— 2015. Effects of Interference Between Selected Loci on the Mutation Load, Inbreeding Depression, and Heterosis. Genetics 201:745+.

Schoen, D., M. Johnston, A. LHeureux, and J. Marsolais, 1997. Evolutionary history of the mating system in Amsinckia (Boraginaceae). Evolution 51:1090-1099.

Shimizu, K. and T. Tsuchimatsu, 2015. Evolution of Selfing: Recurrent Patterns in Molecular Adaptation. Annu Rev Ecol Evol Syst 46:593-622.

Stebbins, G., 1957. Self fertilization and population variability in the higher plants. Am Nat 861:337-354.

Tajima, F., 1989. The effect of change in population size on dna polymorphism. Genetics 123:597-601. URL http://www. genetics.org/content/123/3/597.

Takebayashi, N. and P. L. Morrell, 2001. Is self-fertilization an evolutionary dead end? Revisiting an old hypothesis with genetic theories and a macroevolutionary approach. Am J Bot 88:1143-1150.

Thomann, M., E. Imbert, C. Devaux, and P.-O. Cheptou, 2013. Flowering plants under global pollinator decline. Trends Plant Sci 18:353-359.

Van Daele, I., N. Gonzalez, I. Vercauteren, L. de Smet, D. Inzé, I. RoldánRuiz, and M. Vuylsteke, 2012. A comparative study of seed yield parameters in arabidopsis thaliana mutants and transgenics. Plant Biotechnol J 10:488-500.

Wright, S. I. and S. C. H. Barrett, 2010. The Long-Term Benefits of SelfRejection. Science 330:459-460.

Wright, S. I., S. Kalisz, and T. Slotte, 2013. Evolutionary consequences of self-fertilization in plants. Proc R Soc Lond B Biol Sci 280. 
Wright, S. I., R. W. Ness, J. P. Foxe, and S. C. H. Barrett, 2008. Genomic consequences of outcrossing and selfing in plants. Int J Plant Sci 169:105118.

\section{Tables}

\section{Figure Legends}

Figure 1: Genetic and demographic properties of populations without the evolution of self-fertilisation. a) Observed mean inbreeding depression over all the simulations run (1000) and conditioned on survival (points) in completely outcrossing populations $(\alpha=0)$ as a function of the haploid mutation rate $(U)$ different coefficients of dominance $(h=0,0.1$ and 0.2$)$. Deleterious mutations have coefficients of selection $s=0.02$ (circles) or $s=0.2$ (squares), and $r_{0}=10, K=10000$. Lines represent analytical expectations from Equation (5). b) Mean population size over all the simulations run and conditioned on survival as a function of the self-fertilisation rate. The points are simulation results and the full grey lines the deterministic expectations from Equation (3), replacing $W_{e q}$ with $W_{\text {det }}$ from Equation (4). $r_{0}=4$, $K=10000, s=0.02, h=0.2$.

Figure 2: The demographic and genetic consequences of the evolution of self-fertilisation to an ESS for mildly ( $s=0.02$, grey lines) and strongly ( $s=0.2$, black lines) deleterious mutations in stable populations with $r_{0}=4$, $K=10000, U=0.4, h=0.2$, with a range of the effect of mutations at the modifier set at $d=0.1$ and the mutation rate at the modifier $\mu=10^{-3}$ using results from 1000 simulations run for each parameter set. a) From top to bottom: Changes with time in the mean selfing rate, the mean population size, inbreeding depression over all simulations run conditioned on survival, and the percentage of surviving populations, i.e. simulations that have not (yet) resulted in extinction at a given time. b) The change in mean fitness and inbreeding depression during the evolution of self-fertilisation over all the simulations run and conditioned on survival. The line with the black contour represents analytical expectations using Equations (4) and (5). 
Figure 3: The mean demographic and genetic consequences of the evolution of self-fertilisation (over 1000 independent simulations) with modifiers of strong effect in stable $\left(r_{0}=4\right.$, sub-figures $\left.a, b\right)$ and unstable $\left(r_{0}=2\right.$, subfigures c,d) populations with parameter values $K=10000, U=0.3, h=0.2$, $s=0.02$ and $\mu=10^{-3}$. a) Changes with time in the mean selfing rate, mean population size and inbreeding depression over all simulations run conditioned on survival, as well as the percentage of viable populations, during the evolution of self-fertilisation. $\alpha_{m}=0.3,0.6$ and 0.9 shown respectively by black, dark grey and grey lines. b) The change in mean fitness and inbreeding depression during the evolution of self-fertilisation over all the simulations run and conditioned on survival. The line with the black contour represents analytical expectations using Equations (4) and (5). c) Same as a) but for unstable populations. The dotted line represents the percentage of surviving populations over 1000 simulations in which self-fertilisation does not evolve $(\alpha=0)$. d) Same as b) but with unstable populations.

Figure 4: The mean change in the selfing rate and effective population size relative to demographic size with time over all simulations run (1000 independent runs) and conditioned on survival for $\alpha_{m}=0.3$ (black line) and 0.6 (grey line). $r_{0}=2, K=10000, U=0.3, h=0.2, s=0.02$ and $\mu=10^{-3}$. The dotted lines represent the theoretical expectation of the ratio $N_{e} / N=(2-\alpha) / 2$, where $\alpha$ the selfing rate is replaced by $\alpha_{t}$ in our simulations, therefore taking into account the changing selfing rate with time.

Figure 5: Effect of demographic parameters during the evolution of self-fertilisation (with $\alpha_{m}=0.6$ ) on population size and extinction. In grey $r_{0}=2$ and $K=10000$, in black $r_{0}=4$ and $K=2350$, giving the same initial outcrossing population sizes. $U=0.3, h=0.2, s=0.02$ and $\mu=10^{-3}$. Means obtained from 1000 independent simulations per parameter set and conditioned on survival.

\section{Figures}



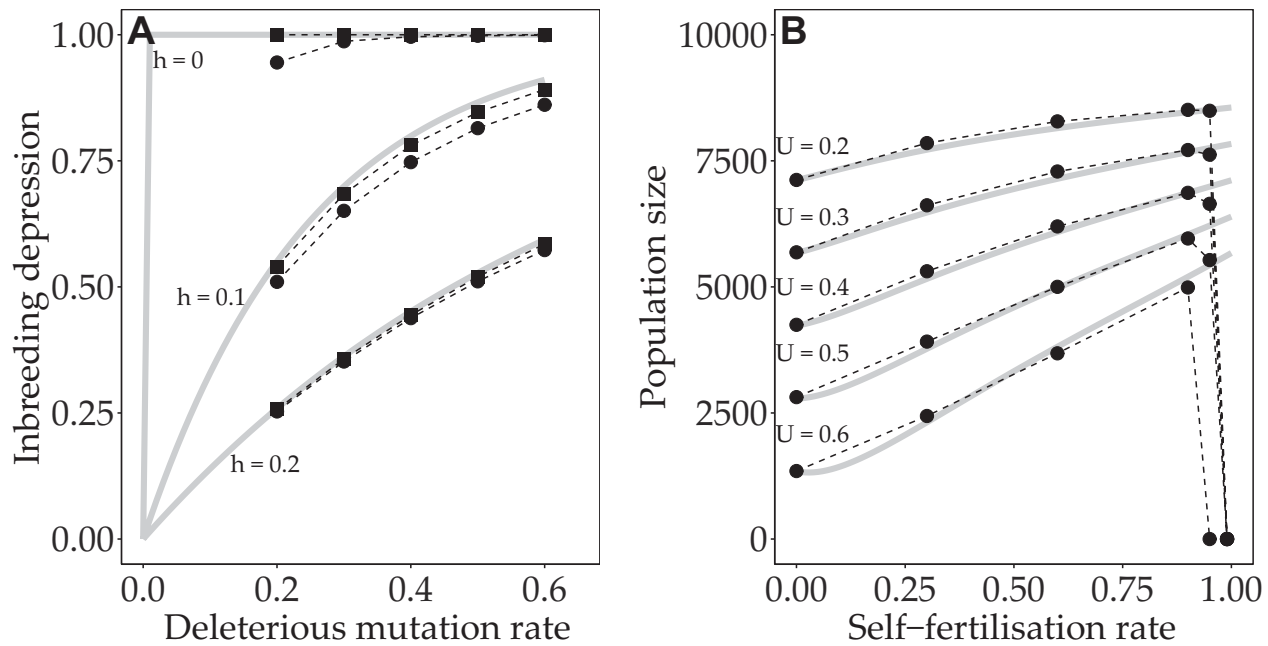

Figure 1: 

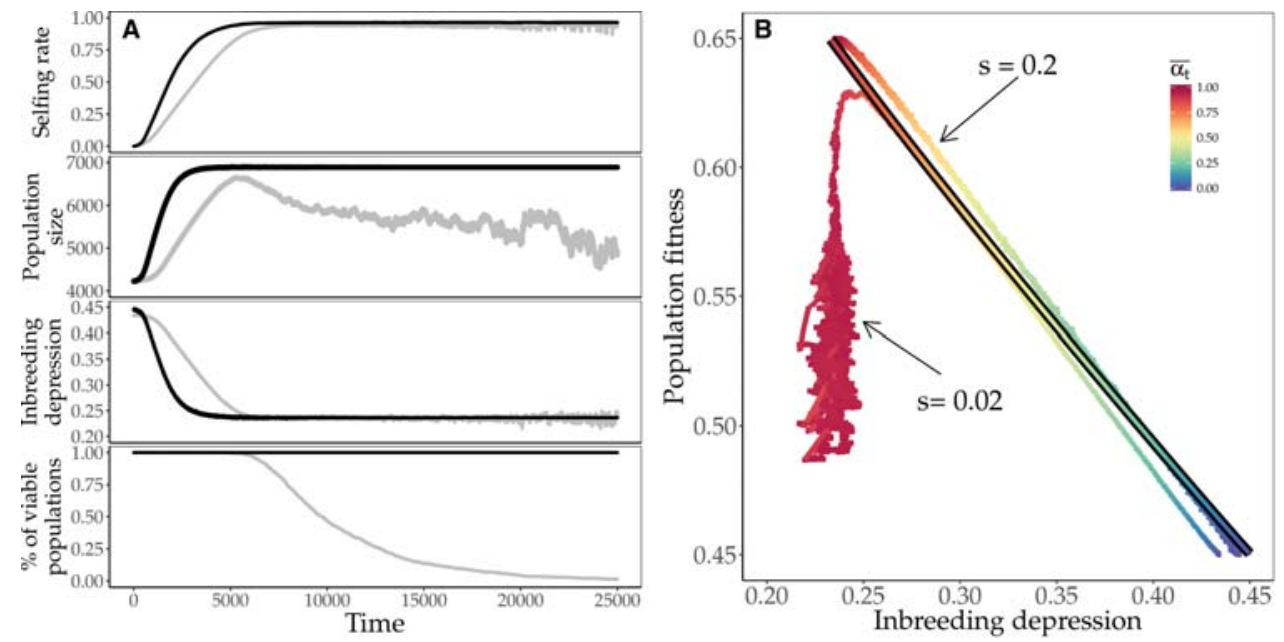

Figure 2: 

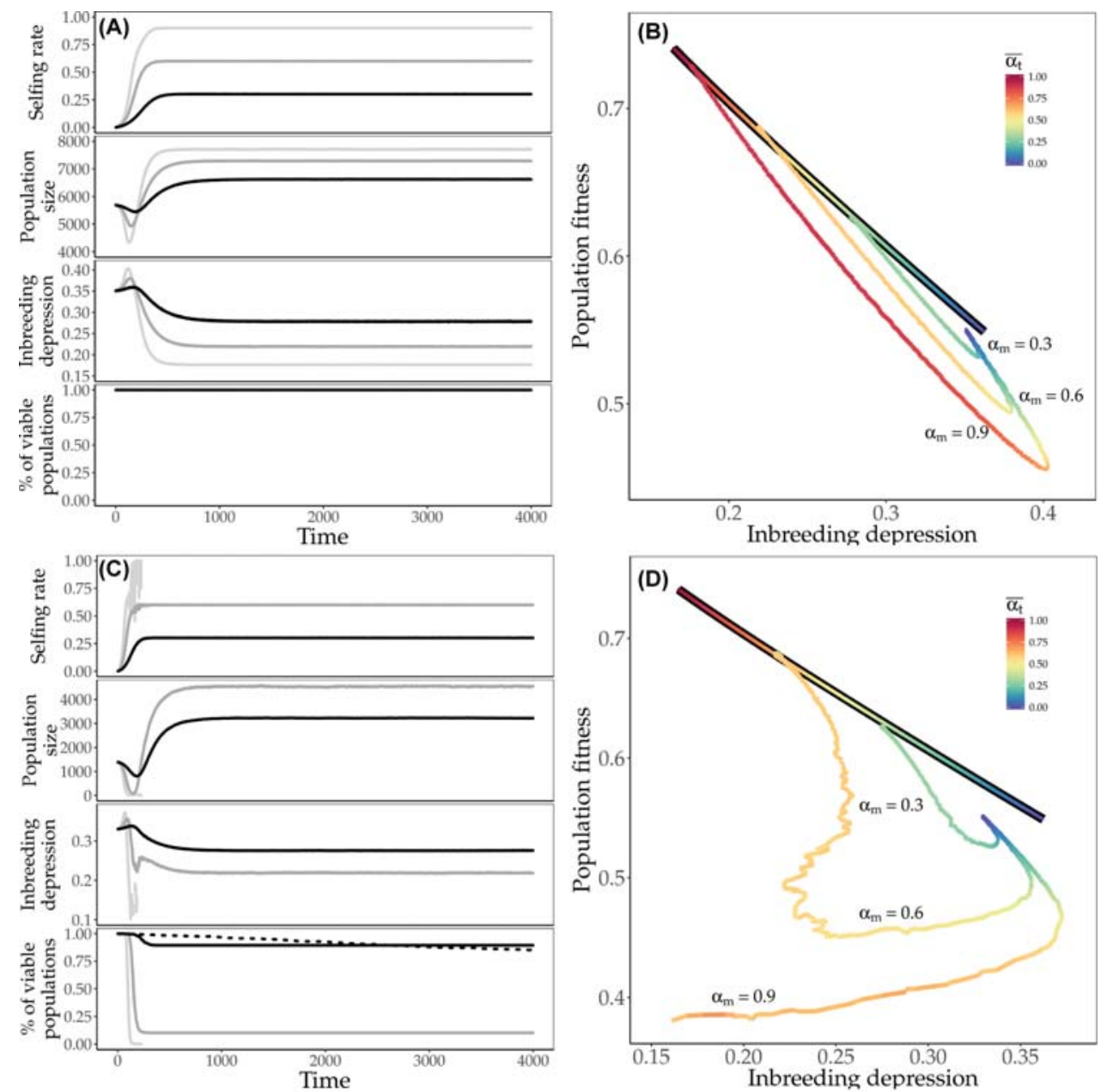

Figure 3: 
Figure 4:

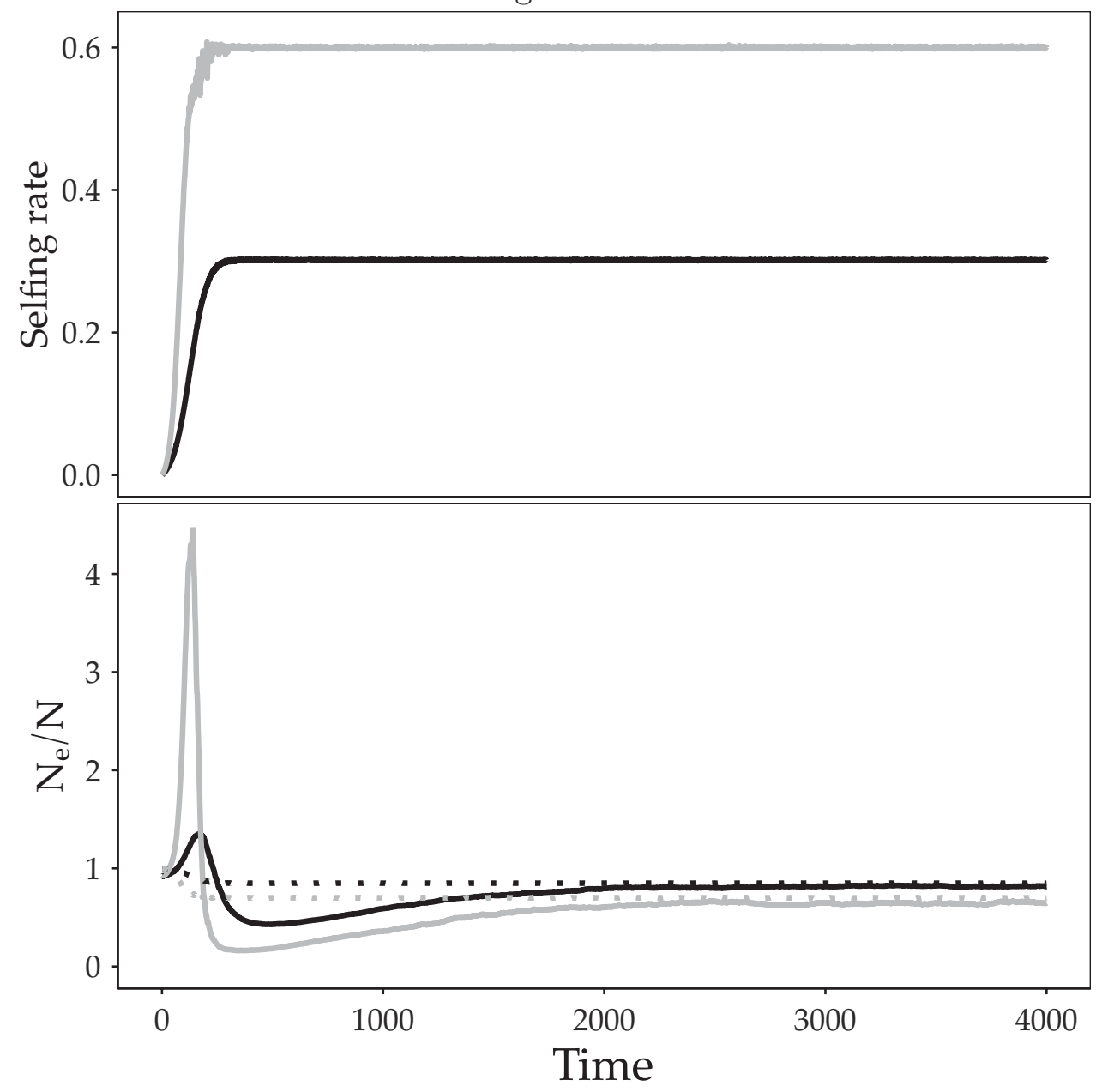


Figure 5:

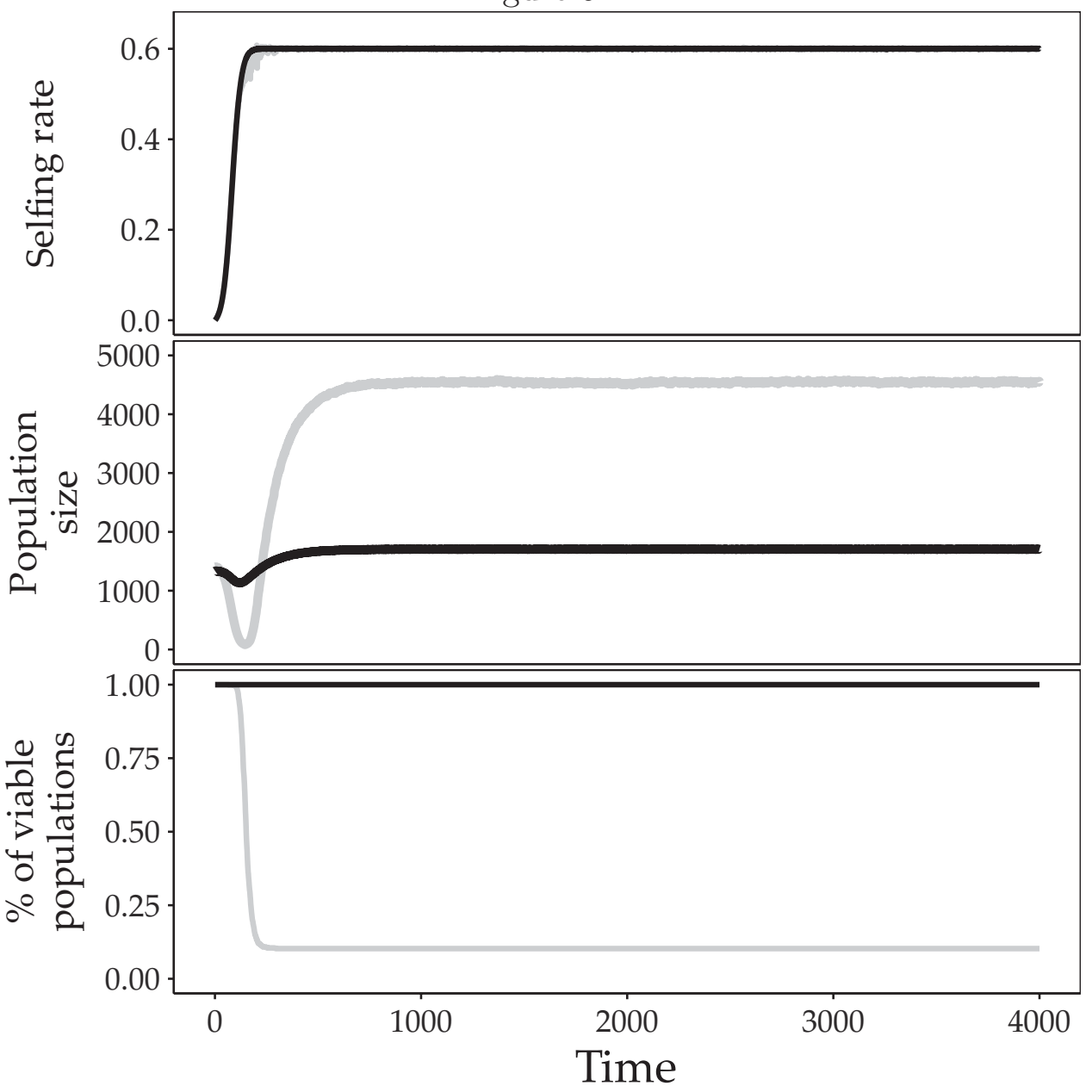




\begin{tabular}{|c|c|c|c|c|}
\hline \multicolumn{2}{|c|}{ Initial statusParameter sets } & \multirow{3}{*}{$\begin{array}{l}\text { Evolution of selfing } \\
\text { Evolution to ESS }\end{array}$} & Final status & Details \\
\hline \multirow[t]{3}{*}{ Stable } & \multirow[t]{3}{*}{ high $r_{0}$ and/or low $U$} & & \multicolumn{2}{|c|}{ Extinction (low $s$ ) Evolutionary suicide } \\
\hline & & & Stable (high $s$ ) & Larger population sizes and higher fitness \\
\hline & & \multicolumn{2}{|c|}{ Modifier of fixed effectStable } & Larger population sizes and higher fitness \\
\hline \multirow[t]{3}{*}{ Unstable } & low $r_{0}$ and/or high $U$ low & $s$ Evolution to ESS & Extinction & Evolutionary suicide \\
\hline & & \multicolumn{2}{|c|}{ Modifier of fixed effectStable } & Evolutionary rescue \\
\hline & & & Extinction & $\begin{array}{l}\text { Demographic consequences of the purge of deleterious mutations } \\
\text { during transition }\end{array}$ \\
\hline
\end{tabular}

Table 1: Summary of simulation results for Stable and Unstable populations depending on the form of evolution of self-fertilisation (continuous or with a single effect modifier). 